\title{
Machine Learning Applications based on SVM Classification: A Review
}

\author{
$1^{\text {st }}$ Dakhaz Mustafa Abdullah \\ Technical College of Informatics, Akre \\ Duhok Polytechnic University \\ Duhok, Iraq \\ dakhaz.abdullah@dpu.edu.kud
}

\author{
$2^{\text {nd }}$ Adnan Mohsin Abdulazeez \\ Research Center of Duhok Polytechnic \\ University \\ Duhok Polytechnic University \\ Duhok, Iraq \\ adnan.mohsin@dpu.edu.krd
}

\begin{abstract}
Extending technologies and data development culminated in the need for quicker and more reliable processing of massive data sets. Machine Learning techniques are used excessively. This paper, therefore, attempts to deal with data processing, using a support vector machine (SVM) algorithm in different fields since it is a reliable, efficient classification method in the area of machine learning. Accordingly, many works have been explored in this paper to cover the use of SVM classifier. Classification based on SVM has been used in many fields like face recognition, diseases diagnostics, text recognition, sentiment analysis, plant disease identification and intrusion detection system for network security application. Based on this study, it can be concluded that SVM classifier has obtained high accuracy results in most of the applications, specifically, for face recognition and diseases identification applications.
\end{abstract}

\section{Keywords-ML, Classification, SVM, Applications.}

\section{INTRODUCTION}

for any action to be carried out by any living being, it is important to have expertise. Each day-to-day task, beginning in the morning, humans easily distinguish the object from another object. Several techniques have been developed to make a computer as capable as human. However, for all types of data set, no single classification approach has been found to provide the best accuracy [1,2]. Machine learning (ML) is defined as a kind of artificial intelligence (AI) that composed of accessible machines with the productivity to be trained without truly programmed [3]. Machine Learning algorithms were used for solving different types of problems in data classification $[4,5,6,7]$. Machine learning technology has grown so quickly in recent years and has been extensively used in many scientific fields $[8,9,10]$. ML algorithms are loosely divided into three groups, namely supervised learning, unsupervised learning and reinforcement learning, as seen in Fig.1. [11,12,13].

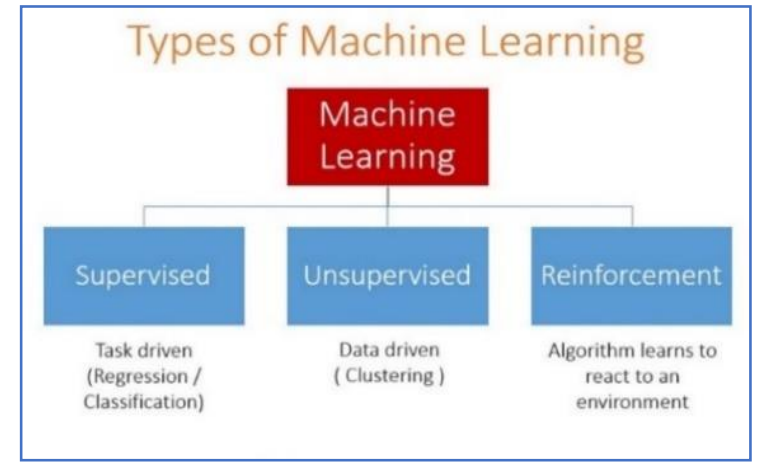

Fig.1. Machine Learning and its Types [8]

There are many good classification techniques in the literature including artificial neural networks, k-nearestneighbors classifier, decision trees, Bayesian classifier and Support Vector Machine (SVM) algorithm. From these techniques, SVM is one of the best-known techniques to optimize the expected solution [14].

SVM algorithm is one of supervised machine learning algorithms based on statistical learning theory [15]. It selects from the training samples a set of characteristic subsets so that the classification of the character subset is equivalent to the division of the entire dataset. the SVM has been used to solve different classification problems successfully in many applications $[16,17]$. So, the application scope is very broad due to its excellent learning ability. For example, intrusion detection, classification of facial expression, prediction of time series, speech recognition, image recognition, signal processing, detection of genes, text classification, recognition of fonts, diagnosis of faults, chemical analysis, recognition of images and other fields. In solving classification problems, the SVM algorithm has certain obvious advantages. It's got a shorter prediction time [18][19]. The accuracy of the target detection classifier can be guaranteed by the global optimal solution. But there are some drawbacks, such as the model of detection is long-established. When processing large-scale data, time complexity and space complexity increase linearly with the increase in data $[20,21]$. In comparison, SVM is more capable of solving smaller samples, nonlinearity and high dimensionality problems compared with other classification algorithms [22,23]. In which case SVM is an 
excellent technique for both linear and non-linear relations modelling. The time for measurement is relatively short compared to other nonparametric methods, such as ANN. Wider training data sets are a challenge when it comes to mechanical training, but SVM continues to generalize itself even with minimal training know-how [24,25].

This paper aims to evaluate SVM's recent applications. This analysis includes articles that used SVM in various fields written from 2016 to 2020. As follows, the remainder of the paper is structured. Section 2 includes a short overview of the SVM. The SVM apps are mentioned in Section 3. A variety of effective SVM implementations for real-world problems are reviewed in Section 4. A discussion and review of SVM implementations is provided in Section 5. a conclusion about the work is provided in Section 6.

\section{SUPPORT VECTOR MACHINE (SVM)}

Vapnik is introduced Support Vector Machine [26,27]. SVM is an algorithm for supervised learning, used for classification and regression [28,29,30]. SVM's aim is to find the individual hyperplane with the highest margin that can divide the classes linearly, as seen in (Fig2). The goal of supporting vector machine learning is to identify data sets where the number of training data is limited and where the optimal solution cannot be ensured by the normal use of large numbers of statistics [31,32,33]

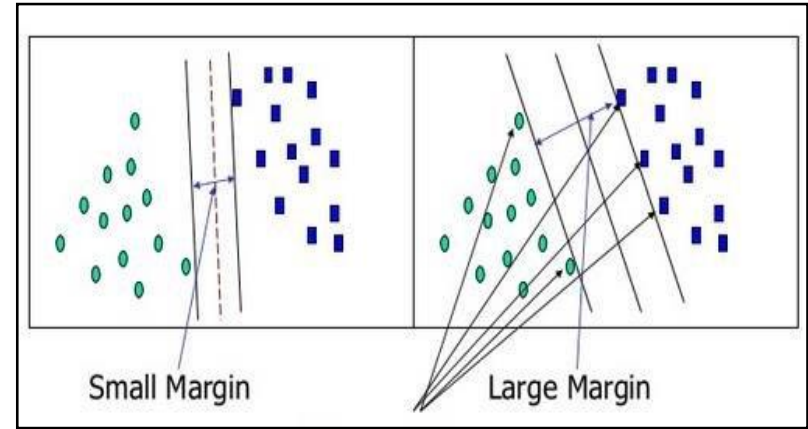

Fig2: Margin and Support Vectors. Left: small margin between 2 classes Right: large margin between 2 classes [31]

In the input space, when the linear separation becomes easier in the kernel functionality, the training data are projected to a feature space of a higher dimension. In order to find a hyperplane that better divides data into its groups, SVM utilizes different kernel functions such as the radial base function (RBF) or polynomial kernel and, when used for minimal training sets, has good classification performance [34]. Therefore, in order to correctly evaluate hyperplanes and reduce classification errors, the SVM algorithm requires a proper kernel function. The fundamental aspect of the SVM technique is the kernel form used.[35,36] The performance of the SVM depends mainly on the size of the kernel, and the smooth surface similarity depends on the higher density of the kernel. [37,38,39]. And unlike some other machine learning methods, the choice of the kernel function is a crucial and demanding operation. The power of the SVM algorithm is therefore the potential to refine itself by modifying its kernel feature and therefore adjusting the dimensionality of the problem to support SVM segregation will also be computationally taxing based on the dimensionality of the problem concerning other algorithms for machine learning [40,41,42].
In many practical fields, many researchers have studied and applied SVM. As the number of training vectors increases, the number of training vectors requiring computing and storage increases, and this is demonstrated in various practical problems beyond their reach $[31,43]$.

\section{APPLICATIONS OF SUPPORT VECTOR MACHINE} (SVM)

The main aim of SVM is to classify unseen data correctly. there are many applications in several fields. SVM applications are:

- Face detection and recognition - parts of the image can be classified using SVM classifier as a face and non-face then creating a square boundary around the face [44-51].

- Disease diagnosis - SVM Classifiers may make a major contribution to the medical field by offering a reliable and rapid diagnosis of diseases. Since the most critical role in the area of health care is the detection of illness. When an infection is detected early, several lives will be spared [52-61].

- Text Recognition - Character recognition in the area of image processing and machine learning has become a difficult and fascinating topic. The most common strategies used in different forms of pattern recognition are SVM. The identification of letters, numbers, figures such as people is never a simple job for a machine. Many techniques in this area were also proposed [62-69].

- Sentiment Analysis Classifications - several types of research have produced forums with SVM Classifier using sentimental analysis where one could easily distinguish optimistic, negative or neutral opinions. The outcome of the opinion analysis is focused on various inquiries such as politics, crime, economics, foreign relations, film, fashion, justice, humanity. Social networking is the primary way of gathering people's thoughts and emotions regarding a different trend. Sentiment analysis is still the most commonly employed study subject [70-77].

- Intrusion Detection System - In the area of information security, identifying and avoiding network intrusion has been an essential matter. The successful method of intrusion detection will reduce the system's false positive rate and increase the accuracy of the classification. Therefore, several SVM-based methods of intrusion detection have been proposed [78-84].

- Identification of plant diseases - Machine learning techniques including (SVM) can be used for the identification of diseases since they only relate to data and prioritize the effects of such activities. This enables diagnosis of the stage of general vegetable diseases [85-89].

\section{LITERATURE REVIEW}

Recently, many works have been implemented using SVM classifier in different fields. So, in this section, some of these works will be elaborated as follows: 


\section{A. Literature on Face Detection and Recognition}

Starting from face detection, many researchers [44-51] implemented algorithms for face detection and recognition. More specifically, Tao et al. [44] and Kumar et al. [45] Proposed face detection techniques based SVM classifier.

Where, Kumar et al. [45] Median filter used for noise reduction in a specific image.by focusing on several facial recognition techniques used for facial identification by the Correlogram, the Discrete Wavelet Transformation (DWT), the Edge Histogram (EH). With the combination of DWT, $\mathrm{EH}$ and Correlogram multi facial detection (MFD), a facial vector is used to identify input faces as single or several, then the SVM classifier. On the standard BAO and Carnegie Mellon University (CMU) datasets, the suggested algorithm was tested, and the precision reached up to 90 percent. From the experiment, it is observed that the SVM classifier more correctly identified the facial images of the previous classifiers. An average precision of identification of 89 percent was obtained for SVM.

Kumar et al [46] proposed automated live facial expression detection (FED) such that support for radial baseline function is used for the extraction and classification of RBF-SVM features by Haar Wavelet Transform (HWT). Genetic algorithm and fuzzy-C-means are used to detect the edges of the face image. The Cohn-Kanade $(\mathrm{CK}+)$ database and the JAFEE facial expression database were included in the experimental results. The other databases used for face recognition are CMU+MIT, LFW-a, FEI and its own site. Finally, $95.6 \%$ and $88.08 \%$ of the CK+ and JAFEE data sets produced a better performance for both datasets. Dino \& Abdulrazzaq [47] implemented another automated framework for the identification of facial expression, which can identify all eight essential facial expressions. The experimental findings revealed that the presented approach offers $93.53 \%$ of the identification rate in the use of the SVM classification, $82.97 \%$ for the use of the MLP classification, and $79.97 \%$ for the KNN classification. The paper concludes that the approach provides stronger outcomes by utilizing the SVM as a classifier.

Azzopardi et al. [48] suggested a system utilizing a fusion of SVM classifiers for gender identification from face pictures. The process, which involves changes in gestures, facial characteristics, context and lighting, was tested on the latest GENDER-FERET dataset. There are different kinds of features in each classifier, including HOG (shape), LBP (texture) and raw pixel values. For the latter functions, we use an SVM with a linear kernel and we use histogram intersection kernel SVMs for the two former ones. The writers come to a consensus by fusing the three classifiers with a plurality vote. In a new dataset that was derived from FERET, they illustrated the feasibility of their method. They reached $92.6 \%$ precision, which exceeded the Face++ and Luxand commercial goods.

Shi et al. [49], Dadi \& Pillutla [50] and Chen \& Haoyu [51] proposed face recognition methods based on SVM. Were, Dadi \& Pillutla [50] for both test and training image extracted Histogram of Oriented Gradient (HOG) features and gave them to the SVM classifier. The Eigen featurebased face recognition algorithm was compared with the algorithm. using 8 different datasets, the suggested algorithm and PCA was verified. The results showed that the proposed algorithm showed a higher face recognition rate in all face datasets compared to the traditional face recognition algorithm based on the Eigen feature. In comparison to the PCA-based face recognition algorithm, there is an 8.75 percent improvement in the face recognition rate. However, Chen \& Haoyu [51] implements an SVM-based facial recognition algorithm in conjunction with the Visual Geometry Community (VGG). The algorithm can not only reliably remove facial characteristics but can also reduce image measurements and avoid irrelevant attributes. the framework of the proposed algorithm can be demonstrated in figure 3 , the. The tests are performed using 400-dimensional feature data on the Labeled Faces in the Wild (LFW) dataset. The results showed that the algorithm proposed reached 97.47 percent, which is the state-of-the-art level.

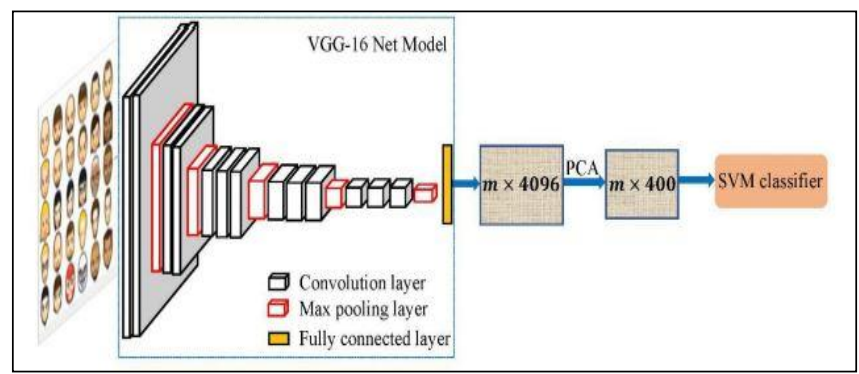

Figure 3: overall framework of the proposed method [51]

\section{B. Literature-based Disease diagnosis applications.}

Regarding the application of diagnosing diseases, various works [52-61] have been implemented using machine learning techniques. Where, techniques of machine learning classification it is very useful in the medical field that provides a quick diagnosis of diseases and accurate, an approach for segmentation of MRI, CT and Ultrasound images was proposed by Asuntha et al. [55] cancer cell identification is performed by analyzing the required characteristics for the two images extracted. To detect the validity of this device, ultrasound images as well. The collection of features is also used with the use of the algorithm of PSO, Genetic Optimization and SVM, giving an accuracy of about 89.5 percent with a reduction in the falsepositive rate. Also, the algorithm that can identify liver Cancer as benign or malignant was implemented by Vadali et al. [56] The project has been tested on a real image of the CT liver. Each module is tested and successfully discovered. SVM is developed and tested to characterize the tumor using known data and irregular obscure data. The consequences of the proposed strategy show that the tumor is accurately recognized and organized as malignant or benign. The accuracy ratio of the framework proposed is 86.7 percent.

Alam et al. [57] have suggested an appropriate algorithm for the identification and prediction of lung cancer using a multiclass SVM classifier. For cancer detection, multi-stage classification has been used. The likelihood of lung cancer can also be predicted by this system. In each phase, enhancement and segmentation of the classification image have been done separately. A 97 percent accuracy for cancer 
identification and 87 percent for cancer prediction was achieved by the proposed algorithm.

Dolatabadi et al. [58], on the other side, suggested a technique for the automated detection of regular and coronary artery disease disorders utilizing electrocardiogramderived heart rate variability (HRV) signal (ECG). The features are obtained in time, frequency and nonlinear domains from the HRV signal. The key component analysis (PCA) is implemented to decrease the size of the extracted properties and to expose the secret details underlaid in the results. In conclusion, using the extracted characteristics, the Vector Classification Support Machine (SVM) was used to classify two classes of data. This thesis optimizes the SVM parameters for enhanced accuracy. The findings revealed a precision of 99.2 percent in detecting normal-class CAD using the proposed algorithm.

Furthermore, Qiao et al.[59] propose a genetic algorithmbased SVM classifier for feature weighting and cataract detection. A cataract is one of the most serious illnesses that can cause blindness. The classifier is applied to the detection and evaluation of cataracts and achieves higher classification accuracy than in previous researches. The practical usage of the enhanced classifier is a major step forward. The algorithm skips the traditional algorithm's redundant measures involving pre-process fundus pictures, extracts and calculates the features directly from original images, as shown in Figure 4. This encourages the precision of the original photos of the fund to

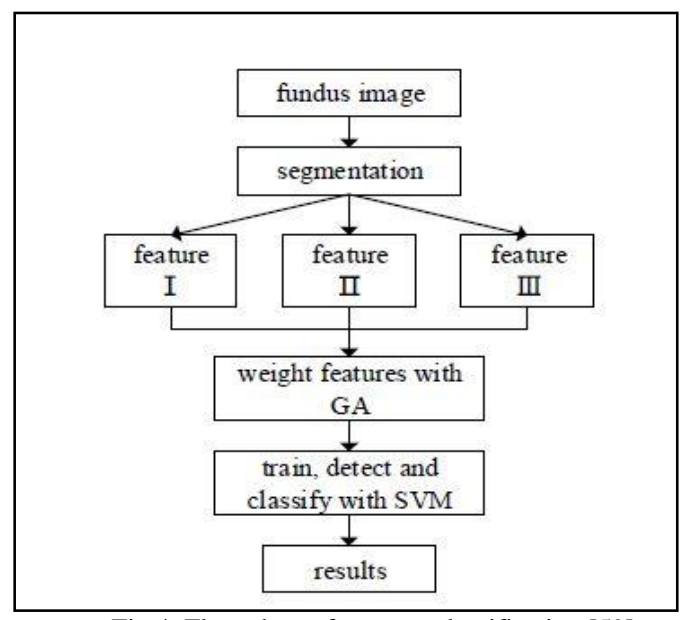

Fig.4: Flow chart of cataract classification [59]

be retained as much as practicable. In order to create the best weight range between the features in the algorithm, the genetic algorithm is used to achieve the best classification result for the classifier.

\section{Literature-based Text Recognition applications}

Text Detection, which has fascinated several researchers[62-69], is another application of SVM. More specifically, Wei et al.[64] proposed utilizing two traditional predictive coding machine learning algorithms: logistic regression (LR) and support vector machines (SVM). The authors carried out tests on the four datasets of actual legal problems to equate deep learning effects with observations obtained using an SVM algorithm. Their results found that with a larger amount of dataset preparation, CNN worked better and could be a suitable method in the text classification of the legal industry. Also, Hassan \& Kadhm [65] introduces an extremely precise handwriting text recognition method. With the SVM polynomial kernel, the computer used 70 percent of the training dataset and 30 percent for research and obtained strong accuracy. There are multiple phases involved in the proposed system: preprocessing, extraction of characteristics, classification and recognition, as seen in Figure 5. The high precision obtained by several variables, starting with the use of the Otsu approach from the effective preprocessing stage, Then with the planned effective segmentation and extraction methods DCT, HOG and finally with a more reliable identification classifier. The system reached 96,317\% highest precision, depending on the methodology and the SVM classification. Besides, Ismayilov [66] suggests another character recognition algorithm focused on the principle of selflearning. The proposed study used the SVM bi-class and multi-class character recognition schemes. Different characteristics are extracted which help to train the proposed system. These features are selected to ensure that the device gains an optimum understanding of the characters and thus achieves high precision. Thus, in addition to the usage of prototypes, the suggested algorithm has the benefit of selflearning for character recognition and thus improves versatility and recognition. In comparison, the findings revealed that the SVM classification for soft features reaches the maximum precision ratio.

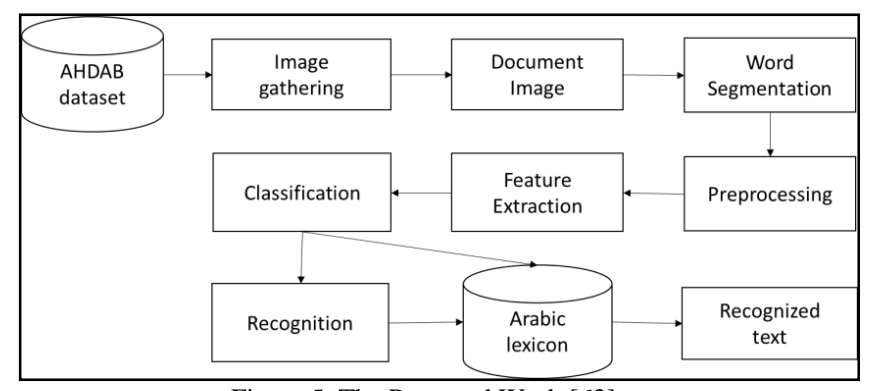

Figure 5. The Proposed Work [63]

Finally, it is simpler for medical practitioners to identify patient symptoms more effectively and to render smart prediction feasible in the future by collecting information from clinical text. Lin et al. [67] suggest a multilabel organized Support Vector Machine (SVM) approach to condition identification. A multi-label approach is implemented that can be used in specific entities identification activities. In which three groups of tests are carried out to test the new model. The results indicate that not only can the disorder mentions in contiguous or discontinuous terms be recognized by the suggested multilabel process, but also mentions the duration of which overlap with each other. In addition, the results found that the proposed SVM hierarchical multi-label model outperformed the random condition field (CRF) model for the disease identification mission.

\section{Literature-based Sentiment analysis applications.}

Sentimental Analysis refers to Natural Language Processing's role of defining if a text contains subjective information and what information it communicates, i.e., whether it is positive, negative or neutral in the attitude behind the text. Many works [67-74] have therefore been proposed for this purpose. More specifically, Wawre et al. [70] Proposed an approach to classifying text feedback on the grounds of the emotions present in such reviews. The 
findings revealed that standard machine learning classification algorithms do not work so well with text sentiment analysis relative to their success with the subjectbased classification. Also, the Naive Bayes Algorithm performed better than SVM Algorithm where the ratios of accuracy for Naive Bayes was $65.57 \%$ while for SVM was $45.71 \%$. Another research has been conducted by Singla et al. [71] for Sentiment analysis of ratings of cell phones and classification of reviews into negative and positive feelings. Three classification models were used to classify reviews after combining the data with an almost equal ratio of positive and negative reviews. On contrary to the results that have been obtained by Wawre et al. [70], the authors Singla et al. [71] proved that predictive accuracy of SVM was better than other classifiers, i.e., Decision Tree and Naïve Bayes. The results accuracy has been cross-validated and among the three models, the maximum precision value was obtained 81.75 percent for SVM.

Also, Rahat et al. [72] used Naïve Bayes and SVM machine learning algorithms for the airline reviews' sentiment classification. The proposed work would be used to carry out an appraisal measure on the comments received from passengers. Good flight is positive sentiment and "Late flight!" is a negative sentiment, as an example. In addition, this positive and negative split statement is used to determine the airline's results. The experimental analysis showed that almost 83 percent accuracy was given by the SVM classifier, which surpassed the Naïve Bayes classifier, which achieved 77 percent accuracy.

Another work for analyzing the sentiments and in opinion mining has been conducted by Bhavitha et al. [73] which also focused on several machine learning techniques. Where the authors presented a detailed survey of different techniques of machine learning and then compared each technique with their accuracy, benefits and limitations. On comparing, they showed that accuracy supervised machine learning technique such as SVM provided accuracy higher than that of unsupervised learning techniques. Another research used automated techniques of text classification by Abro et al. [74] to classify the feedback of the restaurant according to aspect and polarity. Also, this review contrasted two approaches for engineering functions and five ML algorithms to perform three tasks, such as a) classification of restaurant reviews according to the type of entity, b) classification of restaurant reviews according to their characteristics, and c) classification of restaurant reviews according to their polarities.

In addition, the experimental results showed that when used with the SVM algorithm, the word2vec features outperformed other classifiers; NB, LR, RF, and Ensemble by giving task $\mathrm{A}$, task $\mathrm{B}$, and Task $\mathrm{C}$ respectively 76 percent, 72 percent, and 79 percent off overall accuracy.

\section{E. Literature-based Intrusion Detection Techniques.}

The nature of network topology is becoming more complicated with the exponential development of the Internet, rendering it very challenging to monitor network attacks. The traditional solution to passive defence was insufficient to meet the need for network information security. How to successfully track and prevent network interference has been a critical problem in the field of data security. Therefore, many experiments have been carried out to accomplish this goal utilizing machine learning techniques[78-84]. If the successful intrusion detection device is able to reduce the false-positive rate of the system and increase the classification accuracy. The findings obtained with the GF-SVM model increased the overall detection precision. Also, Lei proposes an efficient network anomaly traffic detection algorithm. [78]. Where six types of network features are used in this work, such as Number of sources IP address, Number of the source port, Number of destination IP address, Number of the destination port, Number of types of a packet, and Number of separate packets of the same size as described in Figure 6. The authors then discussed how to generate normalized entropy for the features used in the traffic detection of network anomalies. The network traffic anomaly detection problem is then converted to a classification problem, and to solve it, a hybrid PSO-SVM model is suggested.

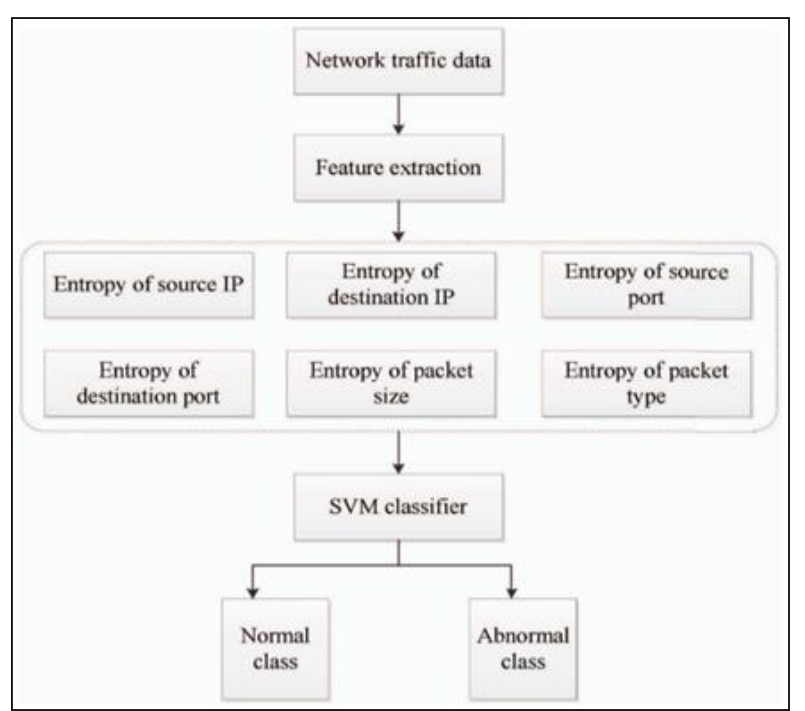

Fig. 6 Framework of the network anomaly traffic detection system [78]

Finally, preliminary findings indicate that different network traffic anomaly patterns may be detected with high precision by the suggested procedure. The machine learning approach is commonly used to avoid phishing attacks. Jupin et al. [79] can thus find four features in the detection of phishing attacks, including URL, domain-based, page-based, and content-based features. Phishing detection may be considered a classification of a data breach, and utilizing ML methods, phishing attacks may be identified. Six widely used methods for ML classification, in which the mechanisms of methods, strengths and weaknesses were discussed, were proposed and examined in detail in this analysis. NB, SVM, DT, RF, k-means and ANN are the chosen techniques. It is challenging to determine the solution is best based on the discussion of the ML approaches, as each strategy has its advantages. The selection of the solution is based upon the dilemma and the functionality chosen since no one method performs well in all situations. As well Emadi \& Mazinani [80] solved another problem of anomaly detection in Wireless Sensor Networks (WSNs) using machine learning techniques. Where, in these networks, the detection of anomalies faces many difficulties. One of these challenges is using a powerful classification algorithm SVM to separate normal data from anomalies. Besides, Network data is 
clustered using the DBSCAN (density-based spatial clustering of noise applications) algorithm. By comparing temperature, humidity, and voltage in different dimensions simultaneously, this approach improved the precision of detection compared with previous methods. Therefore, the advantage of the proposed algorithm over previous algorithms is the use of soft computing techniques, easy implementation, and the simultaneous analysis of these three features to improve detection accuracy.

\section{F. Literature-based plant disease Identification.}

One of the most important concerns of agriculture is pest and disease control in open-air (Arab farming) and greenhouse situations. The most commonly used practice in pest and disease control is to spray pesticides uniformly over the cropping area. This practice has a high financial and significant environmental cost, although it is successful. Residues in crop products, side effects on contamination of groundwater, impacts on local wildlife and ecosystems, and so on, can have environmental impacts. ML is an integrated part of the management of precision farming, where the input of agrochemicals is targeted in terms of time and location. For the detection of diseases, machine learning approaches can be used because they primarily refer to data itself and give preference to the results of some tasks. Therefore, several studies in this area have been carried out [85-89]. More precisely, Shruthi et al. [85] are conducting a comparative analysis on five forms of machine learning classification techniques for plant disease identification. The five forms are SVM, ANN, KNN, CNN, and Fuzzy.
When compared to other classifiers, SVM is used by many authors for disease classification. However, the findings showed that more diseases with high precision were identified by the CNN classifier.

Another plant disease is also Bakana disease or stupid seedling that is a seed-borne rice disease. Infected plants, resulting in a loss of grain yield, can produce or perish empty panicles. Therefore, Chung et al. [86] used a technique to identify contaminated plants that should be screened at the early stages of growth. This work suggests an approach to non-destructively identifying contaminated and healthy seedlings at the age of 3 weeks using machine vision. For the differentiation of infected and healthy seedlings, support vector machine (SVM) classifiers have been developed. In order to pick critical traits and optimal model parameters, a genetic algorithm was used for the SVM classifiers. Infected and stable seedlings were classified by the proposed method with an accuracy of 87.9 percent and a positive predictive value of 91.8 percent.

Ebrahimi et al. [87] suggest a further automatic identification of pests for detecting thrips by utilizing the SVM process on canopy images for crops. Thrips are one of the most dangerous plagues to the greenhouses of strawberry. The SVM approach with differential kernel feature was used for parasite classification and thrip identification. The ratio of major to a small diameter as the geographic index and the Hue, Saturation and Intensification ratios as the color index has been used to construct the SVM layout. Also, a mean square error (MSE), medium square error root (RMSE), a medium absolute error (MAE) and a medium percent error (MPE) were used to determine the classification. The findings demonstrated the strongest classification with less than $2.25 \%$ mean percent error with the SVM-system field index and its intensification as a color index.

\begin{tabular}{|c|c|c|c|c|c|c|}
\hline \multirow{2}{*}{ Ref. } & \multirow{2}{*}{ Years } & \multicolumn{5}{|c|}{ recent works of SVM applications } \\
\hline & & Application & Problems & Methods & Datasets & Results \\
\hline $\begin{array}{c}\text { Kumar et } \\
\text { al.[45] }\end{array}$ & 2019 & Face Detection & $\begin{array}{l}\text { It is not easy for } \\
\text { computer as human } \\
\text { to detect Multiple } \\
\text { faces in a picture. }\end{array}$ & $\begin{array}{l}\text { multiple face detection } \\
\text { technique which used } \\
\text { the Correlogram, } \\
\text { DWT, and EH as well } \\
\text { as SVM Classifier for } \\
\text { face recognition }\end{array}$ & $\begin{array}{l}\text { Two databases, } \\
\text { specifically BAO } \\
\text { database and CMU } \\
\text { database, are utilized } \\
\text { to assess the proposed } \\
\text { framework. }\end{array}$ & $\begin{array}{l}\text { The SVM } \\
\text { classifier } \\
\text { recognized the } \\
\text { facial images } \\
\text { more accurately } \\
\text { from the } \\
\text { previous } \\
\text { classifiers. An } \\
\text { average } \\
\text { recognition } \\
\text { accuracy of } 89 \% \text {. }\end{array}$ \\
\hline $\begin{array}{c}\text { Chen \& } \\
\text { Haoyu [51] }\end{array}$ & 2019 & Face Recognition & $\begin{array}{c}\text { The problem that } \\
\text { the dimension of } \\
\text { facial features is too } \\
\text { large can be solved } \\
\text { using } \\
\text { VGG network } \\
\text { model extracting } \\
\text { facial features that } \\
\text { reduce feature } \\
\text { dimensions. }\end{array}$ & $\begin{array}{c}\text { Face recognition } \\
\text { algorithm based on } \\
\text { SVM combined with } \\
\text { VGG network model } \\
\text { for extracting facial } \\
\text { features. }\end{array}$ & $\begin{array}{c}\text { the algorithm of this } \\
\text { work has been tested } \\
\text { on the CelebA and } \\
\text { LFW datasets }\end{array}$ & $\begin{array}{c}\text { The accuracy of } \\
\text { the proposed } \\
\text { algorithm has } \\
\text { reached } 97.47 \%\end{array}$ \\
\hline
\end{tabular}

TABLE 1: COMPARISON AMONG RECENT WORKS OF SVM APPLICATIONS 


\begin{tabular}{|c|c|c|c|c|c|c|}
\hline $\begin{array}{c}\text { Vadali et al. } \\
{[56]}\end{array}$ & 2019 & $\begin{array}{c}\text { Disease } \\
\text { diagnosis }\end{array}$ & $\begin{array}{c}\text { Liver segmentation and } \\
\text { classification of tumor is } \\
\text { pivotal during treatment } \\
\text { stage. }\end{array}$ & $\begin{array}{c}\text { The proposed } \\
\text { algorithm using } \\
\text { SVM Classifier can } \\
\text { identify the liver } \\
\text { Cancer as benign or } \\
\text { malignant }\end{array}$ & $\begin{array}{l}\text { Image Source for this } \\
\text { work was the UCI } \\
\text { machine learning } \\
\text { repository }\end{array}$ & $\begin{array}{l}\text { The accuracy } \\
\text { rate of the } \\
\text { framework } \\
\text { proposed is } 86.7 \\
\text { percent. }\end{array}$ \\
\hline $\begin{array}{c}\text { Alam et al. } \\
\text { [57] }\end{array}$ & 2018 & $\begin{array}{c}\text { Disease } \\
\text { diagnosis }\end{array}$ & $\begin{array}{c}\text { Recognition and } \\
\text { prediction of lung cancer } \\
\text { in the earliest reference } \\
\text { point stage can be very } \\
\text { useful to improve the } \\
\text { The survival rate of } \\
\text { patients. }\end{array}$ & $\begin{array}{l}\text { Multi-stage SVM } \\
\text { classification for } \\
\text { lung cancer } \\
\text { detection }\end{array}$ & $\begin{array}{l}\text { UCI machine learning } \\
\text { database is used for this } \\
\text { work }\end{array}$ & $\begin{array}{l}\text { The proposed } \\
\text { algorithm } \\
\text { achieved a } \\
\text { precision of } 97 \% \\
\text { for cancer } \\
\text { identification } \\
\text { and } 87 \% \text { for } \\
\text { cancer } \\
\text { prediction. }\end{array}$ \\
\hline Wei et al.[64] & 2018 & $\begin{array}{c}\text { Text } \\
\text { Recognition }\end{array}$ & $\begin{array}{l}\text { Predictive coding can be } \\
\text { used in legal matters to } \\
\text { find relevant or privileged } \\
\text { documents in large sets of } \\
\text { electronically stored } \\
\text { information. }\end{array}$ & $\begin{array}{c}\text { two common } \\
\text { machine learning } \\
\text { algorithms to are } \\
\text { used in predictive } \\
\text { coding; LR and } \\
\text { SVM }\end{array}$ & $\begin{array}{l}\text { SVM algorithm is tested } \\
\text { on the four datasets of } \\
\text { real legal matters. }\end{array}$ & $\begin{array}{l}\text { The findings } \\
\text { showed that } \\
\text { CNN performed } \\
\text { better and it is a } \\
\text { suitable tool for } \\
\text { the legal } \\
\text { industry's text } \\
\text { classification }\end{array}$ \\
\hline Ismayilov [66] & 2018 & $\begin{array}{c}\text { Text } \\
\text { Recognition }\end{array}$ & $\begin{array}{l}\text { Azerbaijani hand-printed } \\
\text { recognition cannot be } \\
\text { considered an 'ended' task } \\
\text { problem. }\end{array}$ & $\begin{array}{c}\text { Hand-printed } \\
\text { character } \\
\text { recognition system } \\
\text { created on the base } \\
\text { of pattern features } \\
\text { and bootstrap } \\
\text { resampling SVM } \\
\text { method. }\end{array}$ & $\begin{array}{l}\text { Recognition database } \\
\text { expressed by Azerbaijani } \\
\text { hand-printed character } \\
\text { examples, digits and } \\
\text { special symbols } \\
\text { is utilized by this work }\end{array}$ & $\begin{array}{l}\text { The results } \\
\text { showed that the } \\
\text { highest accuracy } \\
\text { ratio is obtained } \\
\text { by SVM } \\
\text { classifier using } \\
\text { soft features. }\end{array}$ \\
\hline $\begin{array}{c}\text { Abro et al. } \\
\text { [74] }\end{array}$ & 2020 & $\begin{array}{l}\text { Sentiment } \\
\text { analysis }\end{array}$ & $\begin{array}{l}\text { Regardless of the } \\
\text { extensive amount of work, } \\
\text { it remains difficult to } \\
\text { compare the performance } \\
\text { of these approaches to } \\
\text { classify hotel reviews text. }\end{array}$ & $\begin{array}{l}\text { Automated text } \\
\text { classification } \\
\text { techniques to } \\
\text { classify the } \\
\text { restaurant's reviews } \\
\text { using five ML } \\
\text { Algorithms: SVM, } \\
\text { NB, LR, RF, and } \\
\text { Ensemble classifier. }\end{array}$ & $\begin{array}{l}\text { the SemEval dataset is } \\
\text { utilized for assessing the } \\
\text { five ML Algorithms. }\end{array}$ & $\begin{array}{l}\text { The SVM } \\
\text { algorithm } \\
\text { outperformed by } \\
\text { giving } 76 \% \text {, } \\
72 \%, \text { and } 79 \% \\
\text { off overall } \\
\text { accuracies. }\end{array}$ \\
\hline $\begin{array}{c}\text { Rahat et al. } \\
{[72]}\end{array}$ & 2019 & $\begin{array}{l}\text { Sentiment } \\
\text { analysis }\end{array}$ & $\begin{array}{l}\text { Using sentiment analysis, } \\
\text { it is required to build a } \\
\text { platform where one can } \\
\text { easily identify the } \\
\text { opinions that are either } \\
\text { positive or negative or } \\
\text { neutral. }\end{array}$ & $\begin{array}{l}\text { used Naïve Bayes } \\
\text { and SVM machine } \\
\text { learning algorithms } \\
\text { for the airline } \\
\text { reviews' sentiment } \\
\text { classification }\end{array}$ & $\begin{array}{l}\text { The dataset is collected } \\
\text { from the Review dataset } \\
\text { using Twitter API. }\end{array}$ & $\begin{array}{lr}\text { the } & \text { SVM } \\
\text { classifier, which } \\
\text { surpassed } \\
\text { Naïve } \\
\text { classifier, } \\
\text { achieved } r \\
\text { percent accuracy }\end{array}$ \\
\hline $\begin{array}{c}\text { Jupin et al. } \\
\text { [79] }\end{array}$ & 2019 & $\begin{array}{l}\text { Intrusion } \\
\text { Detection }\end{array}$ & $\begin{array}{l}\text { Phishing detection can be } \\
\text { considered as a } \\
\text { classification of a security } \\
\text { breach. }\end{array}$ & $\begin{array}{c}\text { six popular and } \\
\text { widely used } \\
\text { classification } \\
\text { methods of ML } \\
\text { have been utilized } \\
\text { are; NB, SVM, DT, } \\
\text { RF, k-means } \\
\text { clustering, and ANN }\end{array}$ & $\begin{array}{l}2,000 \text { datasets is used for } \\
\text { testing where } 1,000 \text { URLs } \\
\text { consisted of legitimate } \\
\text { and } 1,000 \text { phishing } \\
\text { URLs. }\end{array}$ & $\begin{array}{l}\text { It is hard to } \\
\text { determine which } \\
\text { method is the } \\
\text { best since there } \\
\text { is no single } \\
\text { method that } \\
\text { works best on } \\
\text { every problem. }\end{array}$ \\
\hline $\begin{array}{c}\text { Emadi \& } \\
\text { Mazinani [80] }\end{array}$ & 2018 & $\begin{array}{l}\text { Intrusion } \\
\text { Detection }\end{array}$ & $\begin{array}{l}\text { the problem of anomaly } \\
\text { detection in Wireless } \\
\text { Sensor Networks (WSNs) }\end{array}$ & $\begin{array}{l}\text { The network data } \\
\text { are clustered using } \\
\text { the DBSCAN } \\
\text { algorithm. It also } \\
\text { analyzes the } \\
\text { accuracy } \\
\text { Using SVM } \\
\text { Classifier }\end{array}$ & $\begin{array}{c}\text { the IRLB } \\
\text { the dataset has been used } \\
\text { by this work }\end{array}$ & $\begin{array}{l}\text { The proposed } \\
\text { method } \\
\text { increased the } \\
\text { accuracy as } \\
\text { compared with } \\
\text { other previous } \\
\text { works. }\end{array}$ \\
\hline $\begin{array}{c}\text { Shruthi et al. } \\
\text { [85] }\end{array}$ & 2019 & $\begin{array}{l}\text { plant disease } \\
\text { Identification }\end{array}$ & $\begin{array}{l}\text { One major effect on low } \\
\text { crop yield is a disease } \\
\text { caused by bacteria, virus } \\
\text { and fungus. which can be } \\
\text { prevented by using plant } \\
\text { diseases detection } \\
\text { techniques }\end{array}$ & $\begin{array}{c}\text { A comparative } \\
\text { study is carried out } \\
\text { on five types of ML: } \\
\text { SVM, ANN, KNN, } \\
\text { CNN, and Fuzzy } \\
\text { classifier }\end{array}$ & $\begin{array}{l}\text { Annotated Dataset which } \\
\text { is a Knowledge based } \\
\text { dataset that is created for } \\
\text { captured images with } \\
\text { different classes are used }\end{array}$ & $\begin{array}{l}\text { The results } \\
\text { showed that } \\
\text { CNN classifier } \\
\text { detected more } \\
\text { diseases with } \\
\text { high accuracy. }\end{array}$ \\
\hline
\end{tabular}




\begin{tabular}{|c|c|c|c|c|c|c|}
\hline $\begin{array}{c}\text { Ebrahimi et al. } \\
{[87]}\end{array}$ & 2017 & $\begin{array}{l}\text { plant disease } \\
\text { Identification }\end{array}$ & $\begin{array}{l}\text { It is necessary to } \\
\text { find a way for } \\
\text { greenhouse } \\
\text { monitoring against } \\
\text { pest attacks. Where, } \\
\text { one of the more } \\
\text { harmful pests that } \\
\text { threaten strawberry } \\
\text { greenhouses is } \\
\text { thrips }\end{array}$ & $\begin{array}{l}\text { Detecting thrips on the } \\
\text { crop canopy images } \\
\text { using SVM } \\
\text { classification method. }\end{array}$ & $\begin{array}{c}\text { Near } 100 \text { sample } \\
\text { images were captured } \\
\text { and obtained from a } \\
\text { strawberry greenhouse } \\
\text { at the Faculty of } \\
\text { Agricultural } \\
\text { Engineering, Tarbiat } \\
\text { Modares University, }\end{array}$ & $\begin{array}{l}\text { Results showed } \\
\text { that using SVM } \\
\text { method made the } \\
\text { best } \\
\text { classification } \\
\text { with MPE of less } \\
\text { than } 2.25 \% \text {. }\end{array}$ \\
\hline
\end{tabular}

\section{DISCUSSION}

It can be observed from the previous section that the researchers have utilized SVM Classifier in different fields. Researchers have identified key points related to their plans' comparison. Table 1 shows a comparison among the investigations mentioned in the previous section. Where the comparison is implemented in terms of Used Methods, problem, application, utilized data set and the main findings.

SVM's have been successfully used to analyze real-world data, often providing better results than other techniques. It can be observed that SVM has played a significant role in the recognition of patterns, which is a highly popular and active research field among researchers. A significant advantage of the SVM method is It typically provides sparse solutions to problems with classification and regression: only a few samples are involved. in deciding the functions of classification or regression. This reality makes it easier to apply SVMs to issues requiring a huge quantity of data, such as processing image, text and tasks in bioinformatics. This has been proved in this study, where, it can be noticed from Table 1 that SVM classifier obtained well results in some applications such as facial recognition, text recognition, and Disease diagnosis in which the highest accuracy obtained in facial recognition application was $97.47 \%$ by [51]. Also, for Disease diagnosis, the best-obtained accuracy was $97 \%$ in cancer identification by [57]. However, for Sentiment analysis the highest obtained accuracy was $77 \%$ by [72] which is not as high as other previous applications. While, for other applications such as intrusion detection applications and plant disease identification, it has been inferred that CNN classifier achieved higher accuracy in detecting diseases than other traditional classifiers.

\section{CONCLUSIONS}

This paper explored active approaches that are implemented using SVM classifier in different research fields. These fields comprise face detection and recognition, Text Recognition, Disease diagnosing, sentiment Analysis, plant disease Identification, Intrusion Detection and many more. Additionally, in all these fields, researchers tried to either use SVM alone for classification or use more than one classification method like NB, LR, RF, and Ensemble classifier in addition to SVM. Where, in most cases, the researchers proved that SVM classifier outperformed other classifiers in terms of Accuracy. Hence, based on our investigation, we can conclude that SVM classifier can be used successfully in most applications especially for the fields of facial recognition in which the highest accuracy obtained was $97.47 \%$ and the same for and diseases diagnostics where the best-obtained accuracy was $97 \%$ in cancer identification. However, for Sentiment analysis the highest obtained accuracy was $77 \%$ which is not as high as other previous applications.

\section{REFERENCES}

[1] Chandra, M. A., \& Bedi, S. S. (2018). Survey on SVM and their application in image classification. International Journal of Information Technology, 1-11

[2] Zeebaree, Diyar Qader, Habibollah Haron, Adnan Mohsin Abdulazeez, and Dilovan Asaad Zebari. "Trainable Model Based on New Uniform LBP Feature to Identify the Risk of the Breast Cancer." In 2019 International Conference on Advanced Science and Engineering (ICOASE), pp. 106-111. IEEE, 2019.

[3] Zeebaree, Diyar Qader, Habibollah Haron, Adnan Mohsin Abdulazeez, and Dilovan Asaad Zebari. "Machine learning and Region Growing for Breast Cancer Segmentation." In 2019 International Conference on Advanced Science and Engineering (ICOASE), pp. 88-93. IEEE, 2019.

[4] Khorshid, S. F., \& Abdulazeez, A. M. (2021). BREAST CANCER DIAGNOSIS BASED ON K-NEAREST NEIGHBORS: A REVIEW. PalArch's Journal of Archaeology of Egypt/Egyptology, 18(4), 19271951.

[5] Zebari, Dilovan Asaad, Diyar Qader Zeebaree, Adnan Mohsin Abdulazeez, Habibollah Haron, and Haza Nuzly Abdull Hamed. "Improved Threshold Based and Trainable Fully Automated Segmentation for Breast Cancer Boundary and Pectoral Muscle in Mammogram Images." IEEE Access 8 (2020): 203097-203116.

[6] Zantalis, F., Koulouras, G., Karabetsos, S., \& Kandris, D. (2019). A review of machine learning and IoT in smart transportation. Future Internet, 11(4), 94

[7] Adnan.M. Abdulazeez, Sulaiman, M. A. Diyar Q.Zeebaree (2020). Evaluating Data Mining Classification Methods Performance in Internet of Things Applications. Journal of Soft Computing and Data Mining, 1(2), 11-25.

[8] Wei, H., Shan, C., Hu, C., Sun, H., \& Lei, M. (2018). Software defect distribution prediction model based on NPE-SVM. China Communications, 15(5), 173-182.

[9] Al-jaboriy, S. S., Sjarif, N. N. A., Chuprat, S., \& Abduallah, W. M. (2019). Acute lymphoblastic leukemia segmentation using local pixel information. Pattern Recognition Letters, 125, 85-90.

[10] Abdullah, D. M., \& Ahmed, N. S. (2021). A Review of most Recent Lung Cancer Detection Techniques using Machine Learning. International Journal of Science and Business, 5(3), 159173.

[11] Jahwar, A. F., \& Abdulazeez, A. M. (2020). Meta-Heuristic Algorithms For K-Means Clustering: A Review. PalArch's Journal of Archaeology of Egypt/Egyptology, 17(7), 12002-12020.

[12] Somvanshi, M., Chavan, P., Tambade, S., \& Shinde, S. V. (2016, August). A review of machine learning techniques using decision tree and support vector machine. In 2016 International Conference on Computing Communication Control and automation (ICCUBEA) (pp. 1-7). IEEE. 
[13] Sulaiman, D. M., Abdulazeez, A. M., Haron, H., \& Sadiq, S. S. (2019, April). Unsupervised Learning Approach-Based New Optimization K-Means Clustering for Finger Vein Image Localization. In 2019 International Conference on Advanced Science and Engineering (ICOASE) (pp. 82-87). IEEE.

[14] Charbuty, B., \& Abdulazeez, A. (2021). Classification Based on Decision Tree Algorithm for Machine Learning. Journal of Applied Science and Technology Trends, 2(01), 20-28.

[15] Wendong, Y., Zhengzheng, L., \& Bo, J. (2017, June). A multi-factor analysis model of quantitative investment based on GA and SVM. In 2017 2nd International Conference on Image, Vision and Computing (ICIVC) (pp. 1152-1155). IEEE.

[16] Kareem, F. Q., \& Abdulazeez, A. M. Ultrasound Medical Images Classification Based on Deep Learning Algorithms: A Review.

[17]- Zeebaree, Diyar Qader, Habibollah Haron, and Adnan Mohsin Abdulazeez. "Gene selection and classification of microarray data using convolutional neural network." In 2018 International Conference on Advanced Science and Engineering (ICOASE), pp. 145-150. IEEE, 2018

[18] Abdulqader, Dildar Masood, Adnan Mohsin Abdulazeez, and Diyar Qader Zeebaree. "Machine Learning Supervised Algorithms of Gene Selection: A Review." Machine Learning 62, no. 03 (2020).

[19] Zebari, R., Abdulazeez, A., Zeebaree, D., Zebari, D., \& Saeed, J. (2020). A Comprehensive Review of Dimensionality Reduction Techniques for Feature Selection and Feature Extraction. Journal of Applied Science and Technology Trends, 1(2), 56-70.

[20] Dai, H. (2018, March). Research on SVM improved algorithm for large data classification. In 2018 IEEE 3rd International Conference on Big Data Analysis (ICBDA) (pp. 181-185). IEEE.

[21] Haji, S. H., \& Abdulazeez, A. M. (2021). COMPARISON OF OPTIMIZATION TECHNIQUES BASED ON GRADIENT DESCENT ALGORITHM: A REVIEW. PalArch's Journal of Archaeology of Egypt/Egyptology, 18(4), 2715-2743.

[22] Tao, P., Sun, Z., \& Sun, Z. (2018). An improved intrusion detection algorithm based on GA and SVM. Ieee Access, 6, 13624-13631.

[23] Chauhan, V. K., Dahiya, K., \& Sharma, A. (2019). Problem formulations and solvers in linear SVM: a review. Artificial Intelligence Review, 52(2), 803-855.

[24] Ademujimi, T. T., Brundage, M. P., \& Prabhu, V. V. (2017, September). A review of current machine learning techniques used in manufacturing diagnosis. In IFIP International Conference on Advances in Production Management Systems (pp. 407-415) Springer, Cham.

[25] D. Q. Zeebaree, A. M. Abdulazeez, D. A. Zebari, H. Haron and H Nuzly, "Multi-level fusion in ultrasound for cancer detection based on uniform lbp features," Computers, Materials \& Continua, vol. 66, no.3, pp. 3363-3382, 2021.

[26] Vapnik, V. N. (1999). An overview of statistical learning theory. IEEE transactions on neural networks, 10(5), 988-999.

[27] Yan, X., \& Jia, M. (2018). A novel optimized SVM classification algorithm with multi-domain feature and its application to fault diagnosis of rolling bearing. Neurocomputing, 313, 47-64.

[28] Wang, C., Zhang, Y., Song, J., Liu, Q., \& Dong, H. (2019). A novel optimized SVM algorithm based on PSO with saturation and mixed time-delays for classification of oil pipeline leak detection. Systems Science \& Control Engineering, 7(1), 75-88.

[29] Fan, M., Wei, L., He, Z., Wei, W., \& Lu, X. (2016). Defect inspection of solder bumps using the scanning acoustic microscopy and fuzzy SVM algorithm. Microelectronics Reliability, 65, 192-197.

[30] Long, S., Huang, X., Chen, Z., Pardhan, S., \& Zheng, D. (2019). Automatic detection of hard exudates in color retinal images using dynamic threshold and SVM classification: algorithm development and evaluation. BioMed research international, 2019

[31] Abd Elkarim, I. S., \& Agbinya, J. (2019). A Review of Parallel Support Vector Machines (PSVMs) for Big Data classification. Australian Journal of Basic and Applied Sciences, 13(12), 61-71.

[32] Li, Q., Du, X., Zhang, H., Li, M., \& Ba, W. (2018, May). Liquid pipeline leakage detection based on moving windows LS-SVM algorithm. In 2018 33rd Youth Academic Annual Conference of Chinese Association of Automation (YAC) (pp. 701-705). IEEE.

[33] Dai, C., Yang, J., Qin, Y., \& Liu, J. (2016, October). Physical layer authentication algorithm based on SVM. In 2016 2nd IEEE
International Conference on Computer and Communications (ICCC) (pp. 1597-1601). IEEE.

[34] Sun, Z., Hu, K., Hu, T., Liu, J., \& Zhu, K. (2018). Fast multi-label low-rank linearized SVM classification algorithm based on approximate extreme points. IEEE Access, 6, 42319-42326.

[35] Batool, M., Jalal, A., \& Kim, K. (2019, August). Sensors Technologies for Human Activity Analysis Based on SVM Optimized by PSO Algorithm. In 2019 International Conference on Applied and Engineering Mathematics (ICAEM) (pp. 145-150). IEEE.

[36] Maulud, D., \& Abdulazeez, A. M. (2020). A Review on Linear Regression Comprehensive in Machine Learning. Journal of Applied Science and Technology Trends, 1(4), 140-147.

[37] Talukdar, S., Singha, P., Mahato, S., Pal, S., Liou, Y. A., \& Rahman, A. (2020). Land-Use Land-Cover Classification by Machine Learning Classifiers for Satellite Observations-A Review. Remote Sensing, 12(7), 1135

[38] Al-Zebari, A., \& Sengur, A. (2019, November). Performance Comparison of Machine Learning Techniques on Diabetes Disease Detection. In 2019 1st International Informatics and Software Engineering Conference (UBMYK) (pp. 1-4). IEEE.

[39] Yi, Q., Wang, H., Guo, R., Li, S., \& Jiang, Y. (2017). Laser ultrasonic quantitative recognition based on wavelet packet fusion algorithm and SVM. Optik, 149, 206-219.

[40] Graser, J., Kauwe, S. K., \& Sparks, T. D. (2018). Machine learning and energy minimization approaches for crystal structure predictions: A review and new horizons. Chemistry of Materials, 30(11), 36013612 .

[41] Khalaf, B. A., Mostafa, S. A., Mustapha, A., Mohammed, M. A., \& Abduallah, W. M. (2019). Comprehensive review of artificial intelligence and statistical approaches in distributed denial of service attack and defense methods. IEEE Access, 7, 51691-51713.

[42] Zhang, Y., Ni, M., Zhang, C., Liang, S., Fang, S., Li, R., \& Tan, Z. (2019, May). Research and application of adaboost algorithm based on svm. In 2019 IEEE 8th Joint International Information Technology and Artificial Intelligence Conference (ITAIC) (pp. 662-666). IEEE.

[43] Dhabhai, A., \& Gupta, Y. K. (2016). Empirical study of image classification techniques to classify the image using svm: A review. International Journal of Innovative Research in Computer and Communication Engineering, 4(10).

[44] Tao, Q. Q., Zhan, S., Li, X. H., \& Kurihara, T. (2016). Robust face detection using local CNN and SVM based on kernel combination. Neurocomputing, 211, 98-105.

[45] Kumar, S., Singh, S., \& Kumar, J. (2019). Multiple face detection using hybrid features with SVM classifier. In Data and Communication Networks (pp. 253-265). Springer, Singapore.

[46] Kumar, S., Singh, S., \& Kumar, J. (2018). Automatic live facial expression detection using genetic algorithm with haar wavelet features and SVM. Wireless Personal Communications, 103(3), 24352453.

[47] Dino, H. I., \& Abdulrazzaq, M. B. (2019, April). Facial expression classification based on SVM, KNN and MLP classifiers. In 2019 International Conference on Advanced Science and Engineering (ICOASE) (pp. 70-75). IEEE.

[48] Azzopardi, G., Greco, A., \& Vento, M. (2016, July). Gender recognition from face images using a fusion of svm classifiers. In International Conference on Image Analysis and Recognition (pp. 533-538). Springer, Cham.

[49] Shi, L., Wang, X., \& Shen, Y. (2020). Research on 3D face recognition method based on LBP and SVM. Optik, 220, 165157.

[50] Dadi, H. S., \& Pillutla, G. M. (2016). Improved face recognition rate using HOG features and SVM classifier. IOSR Journal of Electronics and Communication Engineering, 11(4), 34-44.

[51] Chen, H., \& Haoyu, C. (2019, May). Face Recognition Algorithm Based on VGG Network Model and SVM. In Journal of Physics: Conference Series (Vol. 1229, No. 1, p. 012015). IOP Publishing.

[52] Aljanabi, M., Qutqut, H. M., \& Hijjawi, M. (2018). Machine learning classification techniques for heart disease prediction: A review. International Journal of Engineering \& Technology, 7(4), 5373-5379.

[53] Caballé, N. C., Castillo-Sequera, J. L., Gómez-Pulido, J. A., GómezPulido, J. M., \& Polo-Luque, M. L. (2020). Machine learning applied to diagnosis of human diseases: A systematic review. Applied Sciences, 10(15), 5135 . 
[54] Mahajan, S., Bangar, G., \& Kulkarni, N. (2020). Machine Learning Algorithms for Classification of Various Stages of Alzheimer's Disease: A review. Machine Learning, 7(08).

[55] Asuntha, A., Brindha, A., Indirani, S., \& Srinivasan, A. (2016). Lung cancer detection using SVM algorithm and optimization techniques. J. Chem. Pharm. Sci, 9(4), 3198-3203.

[56] Vadali, S., Deekshitulu, G. V. S. R., \& Murthy, J. V. R. (2019). Analysis of liver cancer using data mining SVM algorithm in MATLAB. In Soft Computing for Problem Solving (pp. 163-175). Springer, Singapore.

[57] Alam, J., Alam, S., \& Hossan, A. (2018, February). Multi-stage lung cancer detection and prediction using multi-class svm classifie. In 2018 International Conference on Computer, Communication, Chemical, Material and Electronic Engineering (IC4ME2) (pp. 1-4). IEEE.

[58] Dolatabadi, A. D., Khadem, S. E. Z., \& Asl, B. M. (2017). Automated diagnosis of coronary artery disease (CAD) patients using optimized SVM. Computer methods and programs in biomedicine, 138, 117126.

[59] Qiao, Z., Zhang, Q., Dong, Y., \& Yang, J. J. (2017, October). Application of SVM based on genetic algorithm in classification of cataract fundus images. In 2017 IEEE International Conference on Imaging Systems and Techniques (IST) (pp. 1-5). IEEE.

[60] Nilashi, M., Ahmadi, N., Samad, S., Shahmoradi, L., Ahmadi, H., Ibrahim, O., ... \& Yadegaridehkordi, E. (2020). Disease Diagnosis Using Machine Learning Techniques: A Review and Classification. Journal of Soft Computing and Decision Support Systems, 7(1), 1930.

[61] Nindrea, R. D., Aryandono, T., Lazuardi, L., \& Dwiprahasto, I. (2018). Diagnostic accuracy of different machine learning algorithms for breast cancer risk calculation: a meta-analysis. Asian Pacific journal of cancer prevention: APJCP, 19(7), 1747.

[62] Li, H. (2020). Text recognition and classification of english teaching content based on SVM. Journal of Intelligent \& Fuzzy Systems, (Preprint), 1-11.

[63] Francis, L. M., \& Sreenath, N. (2020). TEDLESS-Text detection using least-square SVM from natural scene. Journal of King Saud University-Computer and Information Sciences, 32(3), 287-299.

[64] Wei, F., Qin, H., Ye, S., \& Zhao, H. (2018, December). Empirical study of deep learning for text classification in legal document review. In 2018 IEEE International Conference on Big Data (Big Data) (pp. 3317-3320). IEEE.

[65] Hassan, A. K. A., \& Kadhm, M. S. (2016). Arabic handwriting text recognition based on efficient segmentation, DCT and HOG features. International Journal of Multimedia and Ubiquitous Engineering, 11(10), 83-92.

[66] Ismayilov, E. A. (2018). APPLICATION OF SVM AND SOFT FEATURES TO AZERBAIJANI TEXT RECOGNITION. ICTACT Journal on Image \& Video Processing, 9(2).

[67] Lin, W., Ji, D., \& Lu, Y. (2017). Disorder recognition in clinical texts using multi-label structured SVM. BMC bioinformatics, 18(1), 75.

[68] Hassan, A. K. A., Mahdi, B. S., \& Mohammed, A. A. (2019). Arabic handwriting word recognition based on scale invariant feature transform and support vector machine. Iraqi Journal of Science, 381387.

[69] Sharma, S., Sasi, A., \& Cheeran, A. N. (2017, May). A SVM based character recognition system. In 2017 2nd IEEE International Conference on Recent Trends in Electronics, Information \& Communication Technology (RTEICT) (pp. 1703-1707). IEEE.

[70] Wawre, Suchita V., and Sachin N. Deshmukh. (2016)"Sentiment classification using machine learning techniques." International Journal of Science and Research (IJSR) 5.4: 819-821.

[71] Singla, Z., Randhawa, S., \& Jain, S. (2017, June). Sentiment analysis of customer product reviews using machine learning. In 2017 International Conference on Intelligent Computing and Control (I2C2) (pp. 1-5). IEEE.

[72] Rahat, A. M., Kahir, A., \& Masum, A. K. M. (2019, November). Comparison of Naive Bayes and SVM Algorithm based on sentiment analysis using review dataset. In 2019 8th International Conference System Modeling and Advancement in Research Trends (SMART) (pp. 266-270). IEEE.

[73] Bhavitha, B. K., Rodrigues, A. P., \& Chiplunkar, N. N. (2017, March). Comparative study of machine learning techniques in sentimental analysis. In 2017 International Conference on Inventive
Communication and Computational Technologies (ICICCT) (pp. 216221). IEEE.

[74] Abro, S., Shaikh, S., Abro, R. A., Soomro, S. F., \& Malik, H. M. (2020). Aspect Based Sentimental Analysis of Hotel Reviews: A Comparative Study. Sukkur IBA Journal of Computing and Mathematical Sciences, 4(1), 11-20.

[75] Ahmad, M., Aftab, S., Muhammad, S. S., \& Ahmad, S. (2017). Machine learning techniques for sentiment analysis: A review. Int. J. Multidiscip. Sci. Eng, 8(3), 27.

[76] Elmurngi, E., \& Gherbi, A. (2017, August). An empirical study on detecting fake reviews using machine learning techniques. In 2017 seventh international conference on innovative computing technology (INTECH) (pp. 107-114). IEEE.

[77] Polpinij, J., Srikanjanapert, N., \& Sopon, P. (2017, July). Word2Vec approach for sentiment classification relating to hotel reviews. In International Conference on Computing and Information Technology (pp. 308-316). Springer, Cham.

[78] Lei, Y. (2017, October). Network anomaly traffic detection algorithm based on SVM. In 2017 International Conference on Robots \& Intelligent System (ICRIS) (pp. 217-220). IEEE.

[79] Jupin, J. A., Sutikno, T., Ismail, M. A., Mohamad, M. S., Kasim, S., \& Stiawan, D. (2019). Review of the machine learning methods in the classification of phishing attack. Bulletin of Electrical Engineering and Informatics, 8(4), 1545-1555.

[80] Emadi, H. S., \& Mazinani, S. M. (2018). A novel anomaly detection algorithm using DBSCAN and SVM in wireless sensor networks. Wireless Personal Communications, 98(2), 2025-2035.

[81] Gharaee, H., \& Hosseinvand, H. (2016, September). A new feature selection IDS based on genetic algorithm and SVM. In 2016 8th International Symposium on Telecommunications (IST) (pp. 139144). IEEE.

[82] Yavanoglu, O., \& Aydos, M. (2017, December). A review on cyber security datasets for machine learning algorithms. In 2017 IEEE International Conference on Big Data (Big Data) (pp. 2186-2193). IEEE

[83] Wang, C., Zheng, J., \& Li, X. (2017, October). Research on DDoS attacks detection based on RDF-SVM. In 2017 10th International Conference on Intelligent Computation Technology and Automation (ICICTA) (pp. 161-165). IEEE.

[84] Tan, B., Tan, Y., \& Li, Y. X. (2016). Research on Intrusion Detection System Based on Improved PSO-SVM Algorithm. Chemical Engineering Transactions, 51, 583-588.

[85] Shruthi, U., Nagaveni, V., \& Raghavendra, B. K. (2019, March). A review on machine learning classification techniques for plant disease detection. In 2019 5th International Conference on Advanced Computing \& Communication Systems (ICACCS) (pp. 281-284). IEEE.

[86] Chung, C. L., Huang, K. J., Chen, S. Y., Lai, M. H., Chen, Y. C., \& Kuo, Y. F. (2016). Detecting Bakanae disease in rice seedlings by machine vision. Computers and electronics in agriculture, 121, 404411.

[87] Ebrahimi, M. A., Khoshtaghaza, M. H., Minaei, S., \& Jamshidi, B. (2017). Vision-based pest detection based on SVM classification method. Computers and Electronics in Agriculture, 137, 52-58.

[88] Liakos, K. G., Busato, P., Moshou, D., Pearson, S., \& Bochtis, D. (2018). Machine learning in agriculture: A review. Sensors, 18(8), 2674.

[89] Silva, J. C. F., Teixeira, R. M., Silva, F. F., Brommonschenkel, S. H., $\&$ Fontes, E. P. (2019). Machine learning approaches and their current application in plant molecular biology: A systematic review. Plant Science, 284, 37-47 